\title{
Probing Mixing of Photons and Axion-Like Particles by Geometric Phase
}

\author{
A. Capolupo, G. Lambiase, and G. Vitiello \\ Dipartimento di Fisica, "E.R. Caianiello" Università di Salerno, INFN, Gruppo Collegato di Salerno, 84084 Fisciano, Italy
}

Correspondence should be addressed to A. Capolupo; capolupo@sa.infn.it

Received 20 August 2015; Revised 16 October 2015; Accepted 25 October 2015

Academic Editor: Juan José Sanz-Cillero

Copyright (C) 2015 A. Capolupo et al. This is an open access article distributed under the Creative Commons Attribution License, which permits unrestricted use, distribution, and reproduction in any medium, provided the original work is properly cited. The publication of this article was funded by SCOAP

We find that a geometric phase characterizes the phenomenon of mixing of photons with axion-like particles (ALPs). The laboratory observation of such a phase may provide a novel tool able to detect such a mixing phenomenon. We show that the geometric phase is dependent on the axion-like particle mass and coupling constant. We discuss an interferometric experiment able to detect the geometric phase associated with the ALPs-photon mixing.

\section{Introduction}

In the physics beyond the Standard Model much attention is devoted to the study of ultralight and weakly coupled particles. In particular the axion and axion-like particles (ALPs) and their properties have been thoroughly analyzed. The study of ALPs starts with the assumption of the existence of the axion in order to resolve the strong $C P$ problem in quantum chromodynamics $[1,2]$. Such a particle is supposed to be a pseudo-Nambu-Goldstone boson with no electric charge and mass expected to range between $10^{-6}$ and $10^{-2} \mathrm{eV}$, with very small cross section for strong and weak interactions. An interesting property of the axion and of the ALPs is that they can couple to an electromagnetic field, so that they should mix with photons and oscillate in the presence of strong magnetic fields $[3,4]$. Axions and ALPs are proposed to be natural candidates for cold dark matter. Their existence could be revealed in the anomalous cooling of white dwarfs and the anomalous transparency of the Universe for very high energy gamma rays.

Different kinds of terrestrial experiments have attempted to detect axions. The proof of their existence is of course a target of primary experimental importance. A small anomalous rotation of the direction of light polarization, propagating through magnetic fields, has been investigated in PVLAS [5]. In CAST, axions of astrophysical origin are searched by using the Primakoff effect [6-8] and, in ADMX, a strong magnetic field permeating a cold microwave cavity $[9,10]$ has been utilized to reveal the axion presence. The conversion of photons into axions has been also analyzed in a beam of light passing through an intense magnetic field [11-15], although without positive results [11-15]. In cosmology, a recent analysis of axion dark matter scenario has been carried out in [16-18]. It has been shown that the axion and ALP contribution to the dark matter can be obtained provided that the axion mass is $m_{a}=(82.2 \pm 1.1) \mu \mathrm{eV}$ or $m_{a}=(76.6 \pm$ 2.6) $\mu \mathrm{eV}$ (which correspond to the breaking of the PecceiQuinn symmetry at a scale $f_{a}=(7.54 \pm 0.10) \times 10^{10} \mathrm{GeV}$ or $f_{a}=(8.08 \pm 0.27) \times 10^{10} \mathrm{GeV}$, resp. $)$.

In recent years, on the other hand, a great attention has been devoted to the study of topologically nontrivial phases, the so-called geometric phases [19-27]. These are not reducible to or derivable from dynamical phases (neither the vice versa is possible due to their substantial, nonslight, or negligible difference; these ones cannot be written in terms of the other ones) and have been experimentally observed in the evolution of many physical systems, from photons in optical fibers [28] and nuclear magnetic resonance [29] to superconducting circuits [30,31] and electronic harmonic oscillators [32]. Their possible applications in different fields of physics [33-37] have also been studied extensively. Our experience in computing the geometric phase and its consequences in elementary particle physics, in particular in particle mixing 
and oscillations [33, 38, 39], has inspired us to consider the emergence of topological phases in axion physics. Preliminary results have been presented as an extended abstract in the conference report of [40].

In this paper we show that the mixing of photon with ALP gives rise indeed to the appearance of a geometric phase. Specifically, we demonstrate that the geometric phase represents an observable characterization of the time evolution of photons and ALPs only if their mixing is present. On the contrary, no geometric phases arise in the absence of mixing and/or vanishing external magnetic field. In such cases, the total phase is the dynamical one. Therefore, the conclusion is that the geometric phase could be used as a tool to highlight the generation of axions and ALPs by photons propagating through a strong magnetic field. One, indeed, could measure the difference between the geometric phases associated with two rays of light, propagating in two branches of an interferometer, one through a strong magnetic field $B$ and the other one passing through a region, where $B=0$. In particular, we propose considering a laser beam divided by a beam splitter into two rays of equal intensity, one passing in a region permeated by a strong magnetic field and the other acting as a reference beam passing in a region with no magnetic field. The two photon (laser) beams are then recombined together and might interfere. Indeed, in the presence of photon conversion in ALPs, the beam passing through the region with $B \neq 0$ should have a reduced amplitude and a phase shift with respect to the reference beam [41], with the result that there is a reduction in the total intensity of the recombined beam with respect to the original one and an interference pattern due to the difference in the photon phase will appear on the screen.

Here we show that the evolution of photon states, in the presence of magnetic field, contains a phase of geometric origin in addition to the usual dynamical phase derived from Schrödinger's equation. This phase results from the geometrical properties of the parameter space of the Hamiltonian of the system. It is gauge invariant and reparametrization invariant. In the particular case of the mixing of photons with ALPs, the geometric phase depends on the mixing angle $\theta$ and therefore on the coupling constant and ALP mass (see below the expression of the mixing angle).

The beam of photons which propagates with no interaction with the magnetic field has geometric phase equal to zero. The difference between the geometric phases of the beams exiting from the two branches is then the manifestation of the occurrence of the ALP-photon mixing phenomenon in the branch, where $B \neq 0$.

The proposal of such an experiment is new, not drawn from the previous literature. For example, the experiment presented in [42] is based on a different analysis than the one proposed here. In [42] it was proposed to use the interferometer solely to reveal the reduction of the power of the laser beam in the output with respect to the input power, without any consideration concerning the geometrical phases. In the seminal paper [41] the phase in the photonALP mixing in the presence of electromagnetic field has been discussed without reference to the geometric phase, which on the contrary is the main focus of the present paper. Our formalism is fully consistent with the analysis of [41], but it also includes the study of the geometric phase arising as a consequence of the photon-ALP mixing. In the Appendix we outline the main contact points of our formalism with [41].

We observe that the analysis of the geometric phase applies both to scalar and pseudoscalar ALPs and it can be used to discriminate between the two cases. We consider the pseudoscalar case.

The paper is structured as follows. In Section 2 we recall the basic formalism describing the axion-photon mixing. In Section 3 we present the geometric phase characterizing such a phenomenon and study its possible application to the detection of axion and of axion-like particles. Section 4 is devoted to conclusions.

\section{Axion-Photon Mixing}

The lagrangian density of the ALP-photon system is given by $L=L_{\gamma}+L_{a}+L_{\mathrm{QED}}+L_{a \gamma \gamma}$, where $L_{\gamma}=-(1 / 4) F_{\mu \nu} F^{\mu \nu}$ and $L_{a}=$ $(1 / 2)\left(\partial_{\mu} a \partial^{\mu} a-m_{a}^{2} a^{2}\right)$ are the lagrangian densities of free photon and axion; $L_{\mathrm{QED}}=\left(\alpha^{2} / 90 m_{e}^{4}\right)\left[\left(F_{\mu \nu} F^{\mu \nu}\right)^{2}+(7 / 4)\left(F_{\mu \nu} \widetilde{F}^{\mu \nu}\right)^{2}\right]$ is the Heisenberg-Euler term due to loop correction in QED [43-45], which we neglect in the approximation we consider. $L_{a \gamma \gamma}$ is given by

$$
L_{a \gamma \gamma}=\frac{g_{a \gamma \gamma}}{4} a F^{\mu \nu} \widetilde{F}^{\mu \nu},
$$

and it represents the interaction of two photons with the axion pseudoscalar field $a$ in the presence of a magnetic field [46]. Equation (1) can be written as $L_{a \gamma \gamma}=-g_{a \gamma \gamma} a \mathbf{E} \cdot \mathbf{B}$. Here $\widetilde{F}_{\mu \nu}=(1 / 2) \epsilon_{\mu \nu \rho \sigma} F^{\rho \sigma}$ is the dual electromagnetic tensor; $g_{a \gamma \gamma} \equiv g \equiv g_{\gamma} \alpha / \pi f_{a}$ is the axion-photon coupling with dimension of inverse energy, with $g_{\gamma}$ being of the order of the unity, $\alpha=1 / 137$, and $f_{a}$ being the decay constant of ALPs. By means of such an interaction, axion and photon can mix with each other in a background magnetic field.

We consider a monochromatic laser beam splitted into two beams, one propagating through a region permeated by a magnetic field and the other traversing a region with $B=$ 0 . The two beams exiting from the two branches are then recombined together. We assume that the beam propagating in the presence of the magnetic field has the direction along the $z$-axes. Besides the $L_{\mathrm{QED}}$ term, we also neglect the birefringence of fluids in a transverse magnetic field (CottonMouton effect). With these simplifications, choosing the $y$ axis along the projection of $\mathbf{B}$ perpendicular to the $z$-axes, the photon polarization state $\gamma_{x}=\gamma_{\perp}$ decouples and the propagation equations can be written as

$$
\left(\omega-i \partial_{z}+M\right)\left(\begin{array}{c}
\gamma_{\|} \\
a
\end{array}\right)=0
$$

with $\mathbf{B}=\mathbf{B}_{T}$; purely transverse field and mixing matrix $M$ are given by

$$
M=-\frac{1}{2 \omega}\left(\begin{array}{cc}
\omega_{P}^{2} & -g \omega B_{T} \\
-g \omega B_{T} & m_{a}^{2}
\end{array}\right) .
$$


Here $\omega_{P}$ is the plasma frequency and $m_{a}$ is the axion mass. The matrix $M$ can be diagonalized by a rotation to primed fields:

$$
\left(\begin{array}{l}
\gamma_{\|}^{\prime}(z) \\
a^{\prime}(z)
\end{array}\right)=\left(\begin{array}{cc}
\cos \theta & \sin \theta \\
-\sin \theta & \cos \theta
\end{array}\right)\left(\begin{array}{l}
\gamma_{\|}(z) \\
a(z)
\end{array}\right) .
$$

The mixing angle is $\theta=(1 / 2) \arctan \left(2 g \omega B_{T} /\left(m_{a}^{2}-\omega_{P}^{2}\right)\right)$; moreover $\gamma_{\|}^{\prime}(z)=\gamma_{\|}^{\prime}(0) e^{-i \omega_{\gamma} z}, a^{\prime}(z)=a^{\prime}(0) e^{-i \omega_{a} z}$, where

$$
\begin{aligned}
\omega_{\gamma} & =\omega+\Delta_{-}, \\
\omega_{a} & =\omega+\Delta_{+}, \\
\Delta_{ \pm} & =-\frac{\omega_{P}^{2}+m_{a}^{2}}{4 \omega} \pm \frac{1}{4 \omega} \sqrt{\left(\omega_{P}^{2}-m_{a}^{2}\right)^{2}+\left(2 g \omega B_{T}\right)^{2}} .
\end{aligned}
$$

In this way, the photons can be converted into ALPs in the branch of the interferometer in which $B \neq 0$. The conversion of photons in ALPs manifests itself in a reduced amplitude of that beam and in the phase shift with respect to the reference beam.

For the mixing components one has

$$
\left(\begin{array}{l}
\gamma_{\|}(z) \\
a(z)
\end{array}\right)=M(z)\left(\begin{array}{l}
\gamma_{\|}(z) \\
a(z)
\end{array}\right),
$$

where

$$
\begin{aligned}
& M(z) \\
& =\left(\begin{array}{cc}
\cos \theta & -\sin \theta \\
\sin \theta & \cos \theta
\end{array}\right)\left(\begin{array}{cc}
e^{-i \omega_{\gamma} z} & 0 \\
0 & e^{-i \omega_{a} z}
\end{array}\right)\left(\begin{array}{cc}
\cos \theta & \sin \theta \\
-\sin \theta & \cos \theta
\end{array}\right) .
\end{aligned}
$$

Using $(7), \gamma_{\|}(z)$ is obtained as

$$
\begin{aligned}
\gamma_{\|}(z)= & {\left[e^{-i \omega_{\gamma} z} \cos ^{2} \theta+e^{-i \omega_{a} z} \sin ^{2} \theta\right] \gamma_{\|}(0) } \\
& +\cos \theta \sin \theta\left[e^{-i \omega_{\gamma} z}-e^{-i \omega_{a} z}\right] a(0) .
\end{aligned}
$$

Similarly the expression for $a(z)$ is obtained by using (6) and (7). By assuming that the contribution coming from the amplitude $a(0)$ of the axion field is negligible with respect to the $\gamma$-term in the right-hand side of (8), we have

$$
\begin{aligned}
\gamma_{\|}(z) & =M_{11}(z) \gamma_{\|}(0) \\
& =\left[e^{-i \omega_{\gamma} z} \cos ^{2} \theta+e^{-i \omega_{a} z} \sin ^{2} \theta\right] \gamma_{\|}(0) .
\end{aligned}
$$

We observe that the correspondence between the notation of [41] and our notation is $\Delta_{\|}=-\omega_{P} / 2 \omega, \Delta_{a}=-m_{a} / 2 \omega$, and $\Delta_{\|}^{\prime}=\Delta_{+}, \Delta_{a}^{\prime}=\Delta_{-}$. By following [41], we may neglect the phase $e^{-i\left(\omega+\Delta_{\|}\right) z}$ which is common to $\gamma_{\|}(z), \gamma_{\perp}(z)$, and $a_{\|}(z)$; the matrix $M(z)$ becomes

$$
\begin{aligned}
& M(z) \\
& =\left(\begin{array}{cc}
\cos \theta & -\sin \theta \\
\sin \theta & \cos \theta
\end{array}\right)\left(\begin{array}{cc}
e^{-i\left(\Delta_{\|}^{\prime}-\Delta_{\|}\right) z} & 0 \\
0 & e^{-i\left(\Delta_{a}^{\prime}-\Delta_{\|}\right) z}
\end{array}\right)\left(\begin{array}{cc}
\cos \theta & \sin \theta \\
-\sin \theta & \cos \theta
\end{array}\right)
\end{aligned}
$$

and the states (9) are then

$$
\gamma_{\|}(z)=\left[e^{-i\left(\Delta_{\|}^{\prime}-\Delta_{\|}\right) z} \cos ^{2} \theta+e^{-i\left(\Delta_{a}^{\prime}-\Delta_{\|}\right) z} \sin ^{2} \theta\right] \gamma_{\|}(0) .
$$

In the following section we focus our attention on the geometric phase generated in the laser beam which has been propagating through the branch with the magnetic field. The photon beam carrying such a phase, exiting from such a branch, will then interfere with the photon reference beam.

In closing this section, we observe that it has been shown [7] that the transition rate can be enhanced by filling the conversion region with a buffer gas. This induces an effective photon mass $m_{\gamma}$. The plasma frequency $\omega_{P}=\left(4 \pi \alpha N_{e} / m_{e}\right)^{1 / 2}$, where $N_{e}$ denotes the electron density, plays the role of $m_{\gamma}$ [7].

\section{Geometric Phase and Axion-Photon Mixing}

In order to show that a geometric phase arises in the evolution of the ALP-photon system, that is, in the laser beam passing through the magnetic field, we use the definition of Mukunda-Simon (geometric) phase [25]. Let us therefore briefly introduce such a definition.

Such a phase, derived within a kinematical approach, is associated with any open curve of unit vectors in Hilbert space. For a quantum system whose state vector $|\psi(s)\rangle$ belongs to a curve $C$, with $s$ being real parameter such that $s \in\left[s_{1}, s_{2}\right]$, the Mukunda-Simon phase is defined as [25]

$$
\begin{aligned}
\Phi(C)= & \arg \left\langle\psi\left(s_{1}\right) \mid \psi\left(s_{2}\right)\right\rangle \\
& -\mathfrak{J} \int_{s_{1}}^{s_{2}}\langle\psi(s) \mid \dot{\psi}(s)\rangle d s,
\end{aligned}
$$

where the dot denotes the derivative with respect to the real parameter $s$. The right-hand side of (12) is the first term in (2.11) of [25]. In (12), $\arg \left\langle\psi\left(s_{1}\right) \mid \psi\left(s_{2}\right)\right\rangle$ represents the total phase, while $\mathfrak{J} \int_{s_{1}}^{s_{2}}\langle\psi(s) \mid \dot{\psi}(s)\rangle d s$ is the dynamical one. The difference between the two, if not zero, is by definition the geometric phase.

To our knowledge the geometric phase has not been considered in [41] and in other existing literatures relative to axion and ALPs. The definition in (12) can be applied to the photons interacting with the magnetic field. In this case, the geometric phase is given by

$$
\begin{aligned}
\Phi_{\gamma}(z)= & \arg \left\langle\gamma_{\|}(0) \mid \gamma_{\|}(z)\right\rangle \\
& -\Im \int_{0}^{z}\left\langle\gamma_{\|}\left(z^{\prime}\right) \mid \dot{\gamma}_{\|}\left(z^{\prime}\right)\right\rangle d z^{\prime},
\end{aligned}
$$

with $\left|\gamma_{\|}(z)\right\rangle$ being the state of the photons. For $\gamma_{\|}(z)$, given by (11), we obtain

$$
\begin{aligned}
\Phi_{\gamma}(z)= & \arg \left[e^{-i\left(\Delta_{\|}^{\prime}-\Delta_{\|}\right) z} \cos ^{2} \theta+e^{-i\left(\Delta_{a}^{\prime}-\Delta_{\|}\right) z} \sin ^{2} \theta\right] \\
& +\left[\left(\Delta_{\|}^{\prime}-\Delta_{\|}\right) \cos ^{4} \theta+\left(\Delta_{a}^{\prime}-\Delta_{\|}\right) \sin ^{4} \theta\right] z \\
& -\frac{1}{4} \sin ^{2} 2 \theta \cos \left(\frac{\Delta_{\mathrm{osc}} z}{\cos 2 \theta}\right),
\end{aligned}
$$


with $\Delta_{\text {osc }} \equiv \Delta_{\|}-\Delta_{a}$ (as in the notation of [41]). Let us introduce the notation $A=1+\theta^{2}\left(\cos \Delta_{\text {osc }} z-1\right)$ and $B=$ $-\theta^{2}\left(\Delta_{\text {osc }} z-\sin \Delta_{\text {osc }} z\right)$. We then consider (14) at the second order in the mixing angle $\theta$ and obtain

(i) $\Phi_{\gamma}(z)=\frac{\pi}{2}+\theta^{2}\left(\Delta_{\mathrm{osc}} z+\cos \Delta_{\mathrm{osc}} z\right)$,

$$
\text { for } A=0, B>0 \text {, }
$$

(ii) $\Phi_{\gamma}(z)=\frac{3 \pi}{2}+\theta^{2}\left(\Delta_{\mathrm{osc}} z+\cos \Delta_{\mathrm{osc}} z\right)$,

$$
\text { for } A=0, B<0 \text {, }
$$

(iii) $\Phi_{\gamma}(z)=\theta^{2}\left(2 \Delta_{\mathrm{osc}} z-\sin \Delta_{\mathrm{osc}} z+\cos \Delta_{\mathrm{osc}} z\right)$,

for $A>0, B \geq 0$,

(iv) $\Phi_{\gamma}(z)=\theta^{2}\left(2 \Delta_{\mathrm{osc}} z-\sin \Delta_{\mathrm{osc}} z+\cos \Delta_{\mathrm{osc}} z\right)$

$$
+2 \pi, \text { for } A>0, B<0,
$$

(v) $\Phi_{\gamma}(z)=\theta^{2}\left(2 \Delta_{\mathrm{osc}} z-\sin \Delta_{\mathrm{osc}} z+\cos \Delta_{\mathrm{osc}} z\right)$

$$
+\pi, \quad \text { for } A<0, \forall B \text {. }
$$

The nonvanishing value of the geometric phase $\Phi_{\gamma}$ indicates univocally the existence of the ALP-photon mixing phenomenon. Indeed, $\Phi_{\gamma}(z)=0$ when the the magnetic field is zero and the mixing angle $\theta$ is equal to zero. This is the case of the reference beam. See the Appendix for a comparison of (14) with the phase definition in [41].

The geometric phase is thus calculated for the mixed photon state. It can be detected in the end crossing point of the laser beams (in which the magnetic field is zero), by analyzing the interference between the laser beam passing through the magnetic field and the reference beam. Indeed, the geometric phase accumulated by the photons during their paths through the magnetic field survives when the photons exit the magnetic field and interfere with the reference beam.

Notice that the geometric phase can be detected in interferometric experiments when the dynamical phase is much smaller than the geometric one. In general, paths of slightly different lengths can be chosen so that the geometric phase dominates over the dynamical one. In the particular case of the mixing, the phase acquired in the branch with $B \neq$ 0 will have both a geometrical and a dynamical component. The total phase is

$$
\begin{aligned}
\Phi_{\mathrm{TOT}}^{B \neq 0} & (z) \\
= & \Phi_{\gamma}(z) \\
& -\left[\left(\Delta_{\|}^{\prime}-\Delta_{\|}\right) \cos ^{4} \theta+\left(\Delta_{a}^{\prime}-\Delta_{\|}\right) \sin ^{4} \theta\right] z \\
& +\frac{1}{4} \sin ^{2} 2 \theta \cos \left(\frac{\Delta_{\mathrm{osc}} z}{\cos 2 \theta}\right) \\
\simeq & \Phi_{\gamma}(z)+\theta^{2}\left(\Delta_{\mathrm{osc}} z+\cos \Delta_{\mathrm{osc}} z\right),
\end{aligned}
$$

where the second term on the right-hand side is the dynamical phase in the presence of mixing. On the contrary, the phase of the beam passing in the $B=0$ branch contains only the dynamical phase:

$$
\begin{aligned}
\Phi_{\mathrm{TOT}}^{B=0}(z) & =\left(\Delta_{\|}^{\prime}-\Delta_{\|}\right) z=-\frac{\Delta_{\mathrm{osc}} z}{2}\left(1-\frac{1}{\cos 2 \theta}\right) \\
& \simeq \theta^{2} \Delta_{\mathrm{osc}} z .
\end{aligned}
$$

By accurately designing the interferometer, the length of the two branches can be linked by the relation

$$
l_{B=0}=\frac{\Delta_{\mathrm{osc}} l_{B \neq 0}+\cos \left(\Delta_{\mathrm{osc}} l_{B \neq 0}\right)}{\Delta_{\mathrm{osc}}} .
$$

Building the interferometer in this way, once the two laser beams are recombined at the detector, the phase difference will be given by the geometric component. The contributions of the dynamical phases of the two beams, in this case, will indeed compensate each other since $\Delta_{\text {osc }} l_{B=0}=\Delta_{\text {osc }} l_{B \neq 0}+$ $\cos \left(\Delta_{\text {osc }} l_{B \neq 0}\right)$ and the phase difference $\Delta \Phi$ will in practice coincide with the geometric phase $\Phi_{\gamma}$ induced by the mixing; that is, $\Delta \Phi=\Phi_{\gamma}$.

The interferometer can be built in a way that the beams traverse the branches back and forth many times before recombining at the detector. This can be achieved by suitable use of the mirrors. In this way, the phase will add many times. In principle, one can obtain effective branches of arbitrary length. In the particular case in which the photons interact with the magnetic field in a region of length the order of the axion-photon oscillation length $l_{\text {osc }}=2 \pi /\left(\Delta_{\|}-\Delta_{a}\right)$, the geometric phase (14) reduces to the Berry-like phase [19]:

$$
\Phi_{\gamma}\left(z=l_{\text {osc }}\right)=2 \pi \sin ^{2} \theta
$$

Equation (19) can be then rewritten for the $n$-cycle case as

$$
\Phi_{\gamma}\left(n l_{\mathrm{osc}}\right)=2 n \pi \sin ^{2} \theta \approx 2 n \frac{g^{2} B^{2} \omega^{2}}{\left(m_{a}^{2}-\omega_{P}^{2}\right)^{2}} .
$$

A Fabry-Perot cavity can be also used in order to enhance the power of the laser light beam.

For our estimations, we will utilize (14) and use the following values of the parameters entering the above equations: magnetic field $B=10 \mathrm{~T} \simeq 1.95 \times 10^{-15} \mathrm{GeV}^{2}$, energy $\omega=10 \mathrm{eV}$, coupling constant $g \in\left[10^{-16}, 10^{-10}\right] \mathrm{GeV}^{-1}$, axion mass $m_{a} \in\left[10^{-6}, 10^{-2}\right] \mathrm{eV}$, and plasma frequency $\omega_{P}=$ $0.9 m_{a}$.

If the experiment runs for the time interval $t=24$ hours, we can obtain observable values of the phases $\Delta \Phi$ as function of the masses and of $g$. Notice that, as observed in [41], only the photon component of the beam is reflected by mirrors, since any physical mirror is transparent to axions. After $N$ reflections the compound effect is $\phi(L)=N \phi(l)$, with $L=N l$ and $l$ being the distance between the reflecting mirrors [41]. 
By fixing the values of the ALP mass and varying the values of $g$, we obtain the following results for the geometric phase:

(i) $m_{a} \sim 10^{-2} \mathrm{eV}$,

$g \in\left[10^{-11}, 10^{-10}\right] \mathrm{GeV}^{-1} \longrightarrow \Phi_{\gamma}(N l) \in\left[10^{-9}, 10^{-7}\right] \pi$,

(ii) $m_{a} \sim 10^{-3} \mathrm{eV}$,

$g \in\left[10^{-12}, 10^{-10}\right] \mathrm{GeV}^{-1} \longrightarrow \Phi_{\gamma}(N l) \in\left[10^{-8}, 10^{-4}\right] \pi$,

(iii) $m_{a} \sim 10^{-4} \mathrm{eV}$,

$g \in\left[10^{-14}, 10^{-10}\right] \mathrm{GeV}^{-1} \longrightarrow \Phi_{\gamma}(\mathrm{Nl}) \in\left[10^{-8}, 1.6\right] \pi$,

(iv) $m_{a} \sim 10^{-5} \mathrm{eV}$,

$g \in\left[10^{-15}, 10^{-10}\right] \mathrm{GeV}^{-1} \longrightarrow \Phi_{\gamma}(\mathrm{Nl}) \in\left[10^{-6}, 0.9\right] \pi$,

(v) $m_{a} \sim 10^{-6} \mathrm{eV}$,

$g \in\left[10^{-16}, 10^{-10}\right] \mathrm{GeV}^{-1} \longrightarrow \Phi_{\gamma}(N l) \in\left[10^{-4}, 0.6\right] \pi$.

These values of $\Phi_{\gamma}(\mathrm{Nl})$ show that the geometric phase is dependent on the value of the axion mass and on the coupling constant $g$. Such values are detectable; therefore the geometric phase could represent a new tool to reveal the existence of ALPs and also to provide a region of exclusion for the parameter characterizing the ALP physics.

One could wonder that the running time $t=24 \mathrm{~h}$ is too long in order for our system to survive against decoherence phenomenon. However, as in the interferometry experiment proposed by Tam and Yang [42], one may consider running time as long as several days (10 days, $240 \mathrm{~h}$, in [42]) provided that one uses laser beams, as it is indeed in our proposed experiment. On the contrary, this would not be the case by considering noncoherent light, which would be trapped in between mirrors in optical cavity systems for a time interval $\ll 1$ sec.

The present technologies allow for phase measurements within a precision of $\sim 10^{-9}$ [47-49]. Then, the values of the geometric phase here obtained are detectable, provided that sufficient intensity of the signal and signal-to-noise ratio are obtained (see below).

Summing up, the presence of a nontrivial geometric phase of the photon demonstrates the presence of axion-photon mixing; it is highly sensible to the axion mass and $g$ and it allows deriving a region of exclusion for the values of the parameters characterizing the axion physics.

We also observe that the so-called Schottky (or shot) noise, associated with the particle nature of light, affects the sensibility of the interferometer. In the standard case, the shot noise for photons from laser light represents the relative fluctuations in the photon number per unit time and it can be significant when the number of photons is small [50]. The magnitude of the shot noise increases as the square root of the expected number $N$ of photons. The signal-tonoise ratio is given by $r=N / \sqrt{N}=\sqrt{N}$ [50]. In the case of the axion-photon mixing, the probability of their conversion is $(g B L)^{2} / 4$, which is obtained for axion mass much smaller than the energy of photons. The signal-tonoise ratio is then given by $(g B L)^{2} N / 4 \sqrt{N}$. However, in a squeezed state, the number of photons measured per unit time can have fluctuations smaller than the square root of the expected number of photons counted in that period of time [51]. This implies the possibility to increase the sensibility of the interferometer if squeezed light is used in the experiment $[52,53]$.

A nonclassical noise reduction of almost $13 \mathrm{~dB}$ below vacuum noise has been observed [54]. Thus, the squeezed light technique has a great application potential. Injected into an interferometer, the quantum noise reduction corresponding to an increase of a factor 10 in laser light power is possible. The use of Fabry-Perot cavity (FPC) in the arms of the interferometer can further increase the sensitivity of the measurements. We stress that a tipical precision in phase measurement, of the order of $10^{-9}$, does not in general guarantee the detectability of the phase, which also depends on the intensity of the signal and in turn on the (small values of the) mixing angle, that is, on $g$. In this connection, in designing the interferometer, we expect that also in our case the strategy outlined in [42] might be adopted in order to boost the sensitivity; namely, one can incorporate in our setup optical delay lines or Fabry-Perot cavities in order to enhance the signal by a factor given by the number of times the laser beam is reflected back and forth in the FPC. By using the estimation made in [42], one might improve the signal-tonoise ratio even by ten $\mathrm{dB}$ provided that one uses squeezed laser beams as already mentioned above.

\section{Conclusions}

We have shown that the axion-photon mixing exhibits a characterizing geometric phase. The existence of the geometric phase for photons propagating in a magnetic field signals unequivocally the occurrence of the axion-photon mixing and may provide a novel tool in the detection of such a phenomenon. The relation between axion mass and coupling constant could be tested in a dedicated interferometer experiment.

For appropriate values of the photon energies $\omega$ and magnetic fields $B$, the geometric phase could provide a parameter window where searching for ALP contributes to cold dark matter and possible contributions of particle mixing phenomena to the Universe dark energy [55-58].

\section{Appendix}

We compare the geometric phase for mixed photons derived in (14) with the phase presented in [41]. Considering the matrix $(10)$ and assuming $a(0) \approx 0$, the field $\gamma_{\|}(z)$ is given by (9); it is reported here again for convenience (see also (11)):

$$
\begin{aligned}
\gamma_{\|}(z) & =M_{11}(z) \gamma_{\|}(0) \\
& =\left[e^{-i \omega_{\gamma} z} \cos ^{2} \theta+e^{-i \omega_{a} z} \sin ^{2} \theta\right] \gamma_{\|}(0) .
\end{aligned}
$$


By following [59], one can define the phase $\phi(z)$ for photons in the presence of axions as

$$
\phi(z)=-\Im M_{11}(z)=\left[e^{-i \omega_{\gamma} z} \cos ^{2} \theta+e^{-i \omega_{a} z} \sin ^{2} \theta\right],
$$

which for small mixing angle is given by

$$
\phi(z)=\theta^{2}\left(\Delta_{\mathrm{osc}} z-\sin \Delta_{\mathrm{osc}} z\right) .
$$

Such a phase is the one reported in [41] (Equation (22) of [41]). It coincides, up to the second order (for $A>0$ and $B \geq$ 0 , cf. Section 3), with the total phase $\phi_{\text {ТОТ }}(z)=\arg \left\langle\gamma_{\|}(0)\right|$ $\left.\gamma_{\|}(z)\right\rangle=\arg M_{11}(z)$, which is also computed in the present paper. As remarked in the text (cf. Section 3) the geometric phase is by definition the difference between the total phase and the dynamical one and represents a topological invariant; it is invariant under gauge and reparametrization transformations. The phase presented in [41] is thus a different quantity with respect to the geometric phase discussed in the present paper and shown to be nonvanishing.

\section{Conflict of Interests}

The authors declare that there is no conflict of interests regarding the publication of this paper.

\section{Acknowledgments}

Partial financial support from MIUR and INFN is acknowledged. G. Lambiase thanks the ASI (Agenzia Spaziale Italiana) for partial support through the ASI Contract no. I/034/12/0.

\section{References}

[1] R. D. Peccei and H. R. Quinn, "CP conservation in the presence of pseudoparticles," Physical Review Letters, vol. 38, no. 25, pp. 1440-1443, 1997.

[2] R. D. Peccei and H. R. Quinn, "Constraints imposed by CP conservation in the presence of pseudoparticles," Physical Review D, vol. 16, no. 6, pp. 1791-1797, 1977.

[3] G. Raffelt, Stars as Laboratories for Fundamental Physics, University of Chicago Press, Chicago, Ill, USA, 1996.

[4] G. G. Raffelt, "Astrophysical axion bounds," in Axions, Lecture Notes in Physics Vol. 741, pp. 51-71, Springer, Berlin, Germany, 2008.

[5] E. Zavattini, G. Zavattini, G. Ruoso, and et al, "New PVLAS results and limits on magnetically induced optical rotation and ellipticity in vacuum," Physical Review D, vol. 77, Article ID 032006, 2008.

[6] S. Aune, M. Arik, K. Barth, and et al, "Search for sub-eV mass solar axions by the CERN axion solar telescope with ${ }^{3} \mathrm{He}$ buffer gas," Physical Review Letters, vol. 107, no. 26, Article ID 261302, 2011.

[7] K. van Bibber, P. M. McIntyre, D. E. Morris, and G. G. Raffelt, "Design for a practical laboratory detector for solar axions," Physical Review D, vol. 39, no. 8, pp. 2089-2099, 1989.

[8] M. Arik, S. Aune, K. Barth et al., "Search for sub-eV mass solar axions by the CERN axion solar telescope with ${ }^{3}$ He buffer gas," Physical Review Letters, vol. 107, no. 26, Article ID 261302, 2011.
[9] S. J. Asztalos, R. F. Bradley, L. Duffy et al., "Improved rf cavity search for halo axions," Physical Review D, vol. 69, no. 1, Article ID 011101, 2004.

[10] S. J. Asztalos, G. Carosi, C. Hagmann et al., "SQUID-based microwave cavity search for dark-matter axions," Physical Review Letters, vol. 104, no. 4, Article ID 041301, 2010.

[11] R. Cameron, G. Cantatore, A. C. Melissinos et al., "Search for nearly massless, weakly coupled particles by optical techniques," Physical Review D, vol. 47, no. 9, pp. 3707-3725, 1993.

[12] M. Fouche, C. Robilliard, S. Faure et al., "Search for photon oscillations into massive particles," Physical Review D, vol. 78, no. 3, Article ID 032013, 2008.

[13] P. Pugnat, L. Duvillaret, R. Jost et al., "Results from the OSQAR photon-regeneration experiment: no light shining through a wall," Physical Review D, vol. 78, no. 9, Article ID 092003, 2008.

[14] P. Pugnat, R. Ballou, M. Schott et al., "Search for weakly interacting sub-eV particles with the OSQAR laser-based experiment: results and perspectives," The European Physical Journal C, vol. 74, article 3027, 2014.

[15] K. Ehret, M. Frede, S. Ghazaryan et al., "New ALPS results on hidden-sector lightweights," Physics Letters B, vol. 689, no. 4-5, pp. 149-155, 2010.

[16] P. Sikivie and Q. Yang, "Bose-Einstein condensation of dark matter axions," Physical Review Letters, vol. 103, no. 11, Article ID 111301, 2009.

[17] P. Sikivie, “The emerging case for axion dark matter," Physics Letters B, vol. 695, no. 1-4, pp. 22-25, 2011.

[18] E. Di Valentino, E. Giusarma, M. Lattanzi, A. Mel-Chiorri, and O. Mena, "Axion cold dark matter: status after Planck and BICEP2," Physical Review D, vol. 90, no. 4, Article ID 043534, 2014.

[19] M. V. Berry, "Quantal phase factors accompanying adiabatic changes," Proceedings of the Royal Society of London A, vol. 392, pp. 45-57, 1984.

[20] S. Pancharatnam, "Generalized theory of interference, and its applications, part 1. Coherent pencil," Proceedings of the National Academy of Sciences India Section A, vol. 44, pp. 247262, 1956.

[21] A. Shapere and F. Wilczek, Geometric Phases in Physics, World Scientific, Singapore, 1989.

[22] J. C. Garrison and E. M. Wright, "Complex geometrical phases for dissipative systems," Physics Letters A, vol. 128, no. 3-4, pp. 177-181, 1988.

[23] A. K. Pati, "Gauge-invariant reference section and geometric phase," Journal of Physics A: Mathematical and General, vol. 28, no. 7, pp. 2087-2094, 1995.

[24] A. K. Pati, "Geometric aspects of noncyclic quantum evolutions," Physical Review A, vol. 52, no. 4, pp. 2576-2584, 1995.

[25] N. Mukunda and R. Simon, "Quantum kinematic approach to the geometric phase. I. General formalism," Annals of Physics, vol. 228, no. 2, pp. 205-268, 1993.

[26] A. Mostafazadeh, "Noncyclic geometric phase and its nonabelian generalization," Journal of Physics A: Mathematical and General, vol. 32, no. 46, pp. 8157-8171, 1999.

[27] J. Anandan, "Nonadiabatic nonabelian geometric phase," Physics Letters A, vol. 133, no. 4-5, pp. 171-175, 1988.

[28] A. Tomita and R. Y. Chiao, "Observation of berry's topological phase by use of an optical fiber," Physical Review Letters, vol. 57, no. 8, pp. 937-940, 1986. 
[29] J. A. Jones, V. Vedral, A. Ekert, and G. Castagnoli, "Geometric quantum computation using nuclear magnetic resonance," Nature, vol. 403, no. 6772, pp. 869-871, 2000.

[30] P. J. Leek, J. M. Fink, A. Blais et al., “Observation of Berry's phase in a solid-state qubit," Science, vol. 318, no. 5858, pp. 1889-1892, 2007.

[31] M. Neeley, M. Ansmann, R. C. Bialczak et al., "Emulation of a quantum spin with a superconducting phase qudit," Science, vol. 325, no. 5941, pp. 722-725, 2009.

[32] M. Pechal, S. Berger, A. A. Abdumalikov Jr. et al., "Geometric phase and nonadiabatic effects in an electronic harmonic oscillator," Physical Review Letters, vol. 108, no. 17, Article ID 170401, 2012.

[33] A. Capolupo, "Probing CRT violation in meson mixing by a noncyclic phase," Physical Review D, vol. 84, no. 11, Article ID 116002, 2011.

[34] A. Capolupo and G. Vitiello, "Spontaneous supersymmetry breaking probed by geometric invariants," Advances in High Energy Physics, vol. 2013, Article ID 850395, 5 pages, 2013.

[35] A. Capolupo and G. Vitiello, "Probing Hawking and Unruh effects and quantum field theory in curved space by geometric invariants," Physical Review D, vol. 88, no. 2, Article ID 024027, 2013.

[36] A. Capolupo and G. Vitiello, "Vacuum condensate, geometric phase, unruh effect, and temperature measurement," Advances in High Energy Physics, vol. 2015, Article ID 878043, 8 pages, 2015.

[37] J. Hu and H. Yu, "Geometric phase for an accelerated two-level atom and the Unruh effect," Physical Review A, vol. 85, no. 3, Article ID 032105, 2012.

[38] M. Blasone, P. A. Henning, and G. Vitiello, "Berry phase for oscillating neutrinos," Physics Letters B, vol. 466, no. 2-4, pp. 262-266, 1999.

[39] M. Blasone, A. Capolupo, E. Celeghini, and G. Vitiello, "Noncyclic phases for neutrino oscillations in quantum field theory," Physics Letters B, vol. 674, no. 1, pp. 73-79, 2009.

[40] A. Capolupo, G. Lambiase, and G. Vitiello, "Axion-photon mixing and geometric phase," Journal of Physics: Conference Series, vol. 626, no. 1, Article ID 012059, 2015.

[41] G. Raffelt and L. Stodolsky, "Mixing of the photon with lowmass particles," Physical Review D, vol. 37, no. 5, pp. 1237-1249, 1988.

[42] H. Tam and Q. Yang, "Production and detection of axion-like particles by interferometry," Physics Letters B, vol. 716, no. 3-5, pp. 435-440, 2012.

[43] W. Heisenberg and H. Euler, "Folgerungen aus der diracschen theorie des positrons," Zeitschrift für Physik, vol. 98, no. 11, pp. 714-732, 1936.

[44] J. S. Schwinger, "On gauge invariance and vacuum polarization," Physical Review, vol. 82, no. 5, pp. 664-679, 1951.

[45] B. Döbrich and H. Gies, "Interferometry of light propagation in pulsed fields," Europhysics Letters, vol. 87, no. 2, Article ID 21002, 2009.

[46] K. A. Olive, K. Agashe, C. Amsler et al., "Review of particle physics," Chinese Physics C, vol. 38, no. 9, Article ID 090001, 2014.

[47] Z. Y. Ou, "Fundamental quantum limit in precision phase measurement," Physical Review A, vol. 55, no. 4, pp. 2598-2609, 1997.

[48] F. W. Sun, B. H. Liu, Y. X. Gong, Y. F. Huang, Z. Y. Ou, and G. C. Guo, "Experimental demonstration of phase measurement precision beating standard quantum limit by projection measurement," Europhysics Letters, vol. 82, no. 2, Article ID 24001, 2008.

[49] Y. Gao and H. Lee, "Sub-shot-noise quantum optical interferometry: a comparison of entangled state performance within a unified measurement scheme," Journal of Modern Optics, vol. 55, no. 19-20, pp. 3319-3327, 2008.

[50] W. Schottky, "Über spontane Stromschwankungen in verschiedenen Elektrizitätsleitern," Annalen der Physik, vol. 362, no. 23 , pp. 541-567, 1918.

[51] C. M. Caves, "Quantum-mechanical noise in an interferometer," Physical Review D, vol. 23, no. 8, pp. 1693-1708, 1981.

[52] The LIGO Scientific Collaboration, "A gravitational wave observatory operating beyond the quantum shot-noise limit," Nature Physics, vol. 7, no. 12, pp. 962-965, 2011.

[53] M. D. Lang and C. M. Caves, "Optimal quantum-enhanced interferometry using a laser power source," Physical Review Letters, vol. 111, no. 17, Article ID 173601, 2013.

[54] T. Eberle, S. Steinlechner, J. Bauchrowitz et al., "Quantum enhancement of the zero-area sagnac interferometer topology for gravitational wave detection," Physical Review Letters, vol. 104, no. 25, Article ID 251102, 2010.

[55] A. Capolupo, S. Capozziello, and G. Vitiello, "Neutrino mixing as a source of dark energy," Physics Letters A, vol. 363, no. 1-2, pp. 53-56, 2007.

[56] A. Capolupo, S. Capozziello, and G. Vitiello, "Dark energy and particle mixing," Physics Letters A, vol. 373, no. 6, pp. 601-610, 2009.

[57] A. Capolupo, S. Capozziello, and G. Vitiello, "Dark energy, cosmological constant and neutrino mixing," International Journal of Modern Physics A, vol. 23, no. 31, pp. 4979-4990, 2008.

[58] M. Blasone, A. Capolupo, S. Capozziello, S. Carloni, and G. Vitiello, "Neutrino mixing contribution to the cosmological constant," Physics Letters, Section A: General, Atomic and Solid State Physics, vol. 323, no. 3-4, pp. 182-189, 2004.

[59] L. Maiani, R. Petronzio, and E. Zavattini, "Effects of nearly massless, spin-zero particles on light propagation in a magnetic field," Physics Letters B, vol. 175, no. 3, pp. 359-363, 1986. 

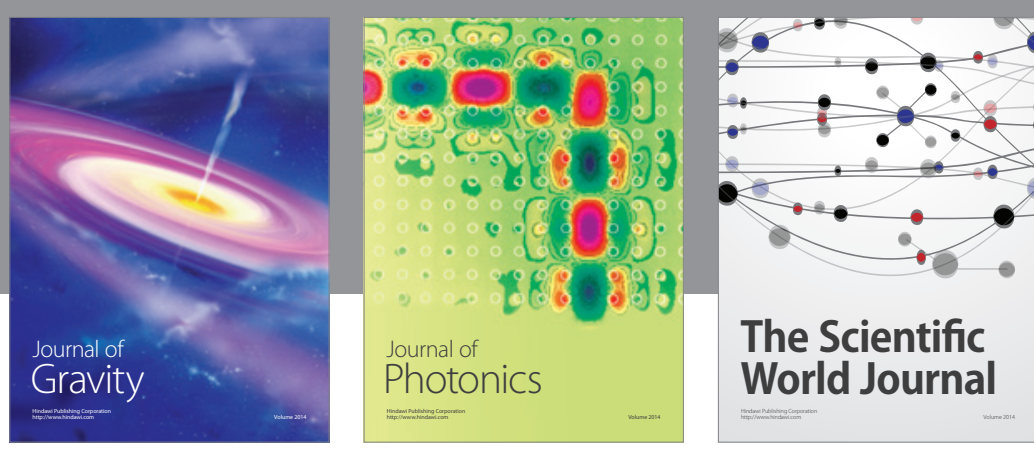

The Scientific World Journal
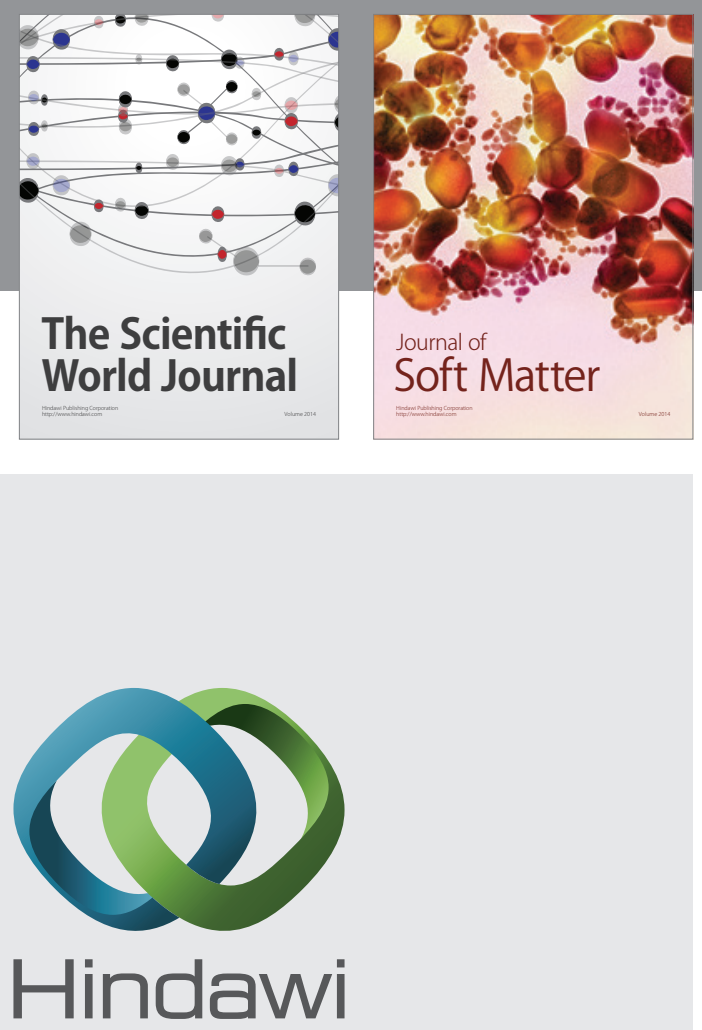

Submit your manuscripts at

http://www.hindawi.com

nternational Journal of

Statistical Mechanics
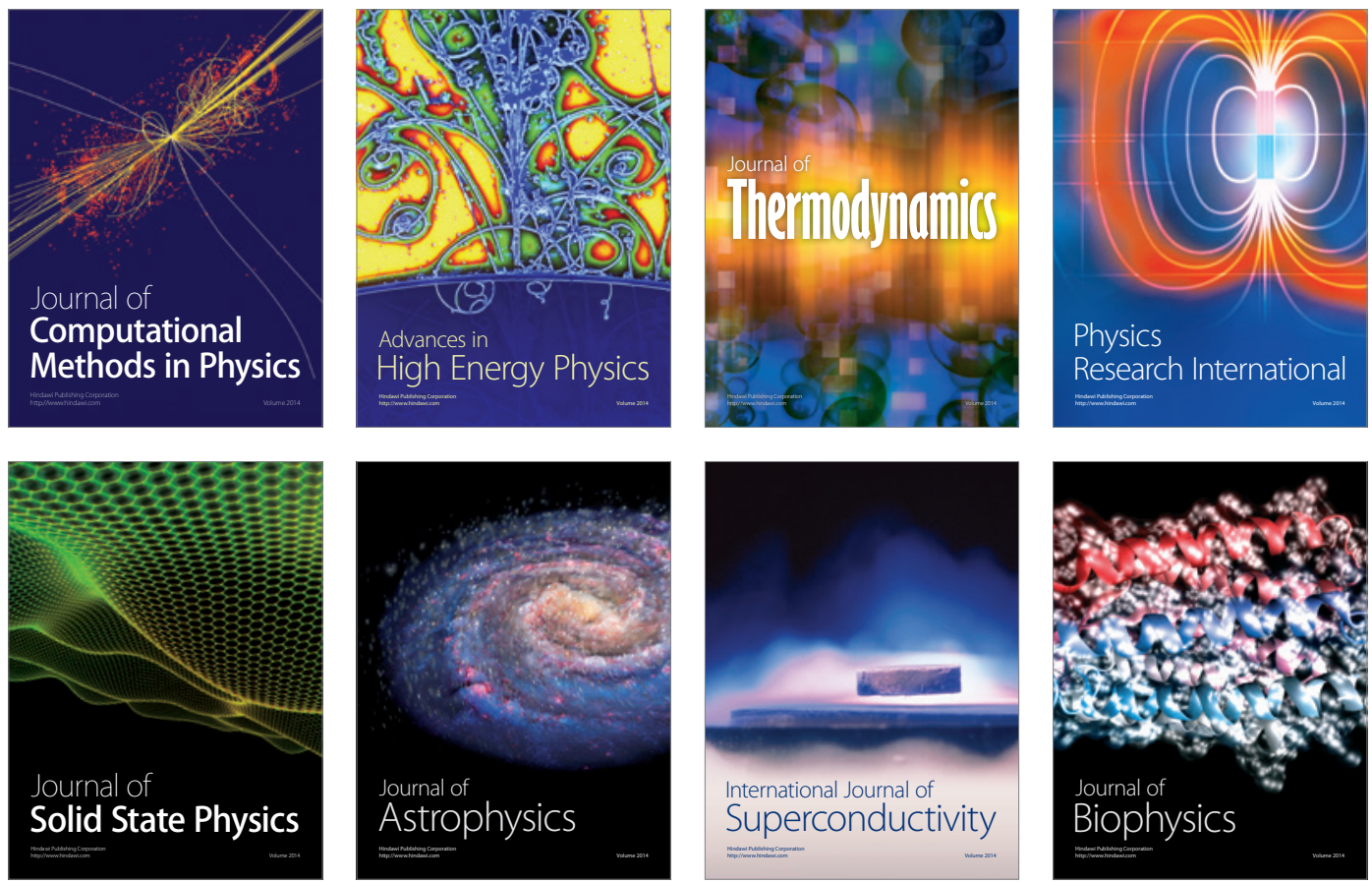
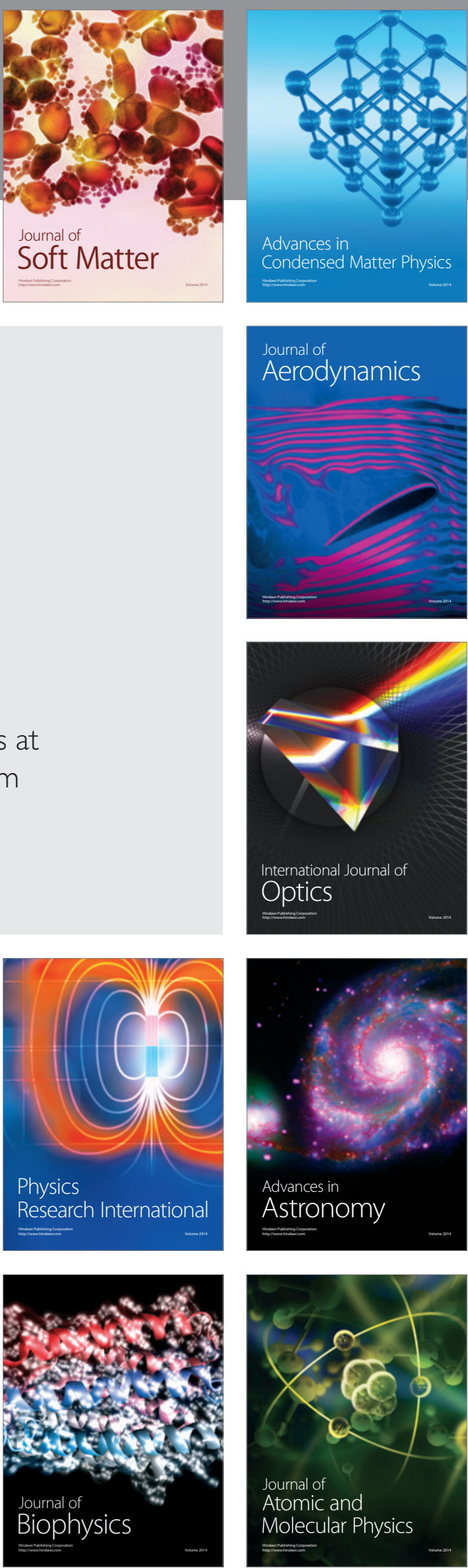